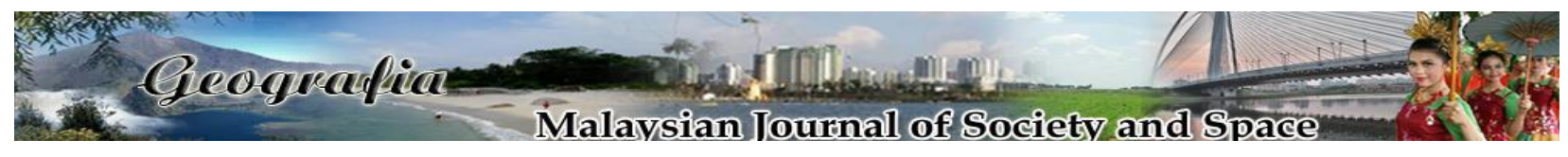

\title{
Low altitude multispectral mapping for road defect detection
}

\author{
Shahrul Nizan Abd Mukti ${ }^{1}$, Khairul Nizam Tahar ${ }^{2}$ \\ ${ }^{1}$ Dewan Bandaraya Kuala Lumpur (DBKL), Department of Infrastructure Planning, Kuala Lumpur \\ ${ }^{2}$ Centre of Studies for Surveying and Geomatics, Faculty of Architecture, Planning and Surveying, \\ Universiti Teknologi MARA, Shah Alam \\ Correspondence: Khairul Nizam Tahar (email: khairu10127@uitm.edu.my)
}

Received: 04 January 2021; Accepted: 22 April 2021; Published: 29 May 2021

\begin{abstract}
Pothole's defect is major damages indicated the road condition visually, and the structural defects due to some potential causes. Nowadays, new forms of remote sensing technique were widely used, but less studies in the application of low altitude multispectral mapping. The potential of multispectral images is its help better in resolution due to its spectral characteristic. Hence, it helps a lot in feature classification with proper training sample, classifier used, and spectral band composite. Thus, this study aims to extract the defective roads by using the multispectral image of Parrot Sequoia with low flight altitude. This study tries to detect a pothole's existence from band combination and supervised classification other than its common use which ultimately for agriculture purposes. The classifier used in this is Maximum Likelihood, Support vector machine (SVM) and Mahalanobis Distance. 15 different probability of band stacks of green, NIR, red edge, and red band were used as multispectral images. The comparison of the performance between the types of classifier and band combination was modeled and discussed in this study. Classifier algorithm maximum likelihood gives the lowest error of $0.108 \mathrm{~m}^{2}$ with a combination of $\mathrm{NIR}+$ red edge band. SVM gives the lowest error of $0.427 \mathrm{~m}^{2}$ with a combination of green $+\mathrm{NIR}+$ red edge + red band. While Mahalanobis distance gives the lowest error of $-0.082 \mathrm{~m}^{2}$ with a combination of red edge + red band. Averagely, Mahalanobis distance gives the lowest error of $0.299 \mathrm{~m}^{2}$ of all bands used.
\end{abstract}

Keywords: Road, pothole, UAV, multispectral, UAV and detection

\section{Introduction}

The integration of geospatial tools and techniques in transportation management is a growing research agenda. The source of data for defect detection varies. The educational or practical background about remote sensing techniques in road maintenance applications is mounting. Roads need well maintain and repair as they indicate an issue of structural adequacy, skid resistance, or surface defect (Schnebele et al., 2015). Some of these indications suitable for remote sensing 
assessment. Hereby define remote sensing as any surveying method that does not require physical contact with the road surface or subsurface. This conventional method today has taken by the new technique of defect detection. A conventional method for road detection required several technicians for manual data collection and many working hours for a rough estimation of damages on the roads. Related information gathered is important for planning and budget preparation (Akagic et al., 2018). At glance, the current remote sensing method used has different platform, sensor, and interest defect features. The various platform uses in remote sensing methods such as satellite (Zhang et al., 2018), unmanned aerial vehicle (UAV) (Iseli \& Lucieer, 2019), airplane and vehicle. Any sensor can fit a different platform if a proper bracket installs to secure the sensor attached to the platform. Sensor use can be any, such as red, green, and blue (RGB) camera, ground-penetrating radar (GPR) (Wolf et al., 2018), infrared (IR) thermography (Gandhi et al., 2019), light detection, and ranging (LIDAR), multispectral (Noi \& Kappas, 2018) and hyperspectral camera. Each detection to the defect road can be evaluate based quantitatively. Different types of road defects happen due to the failure of structural integrity. Types of road defects are cracking the surface, cracking fatigue, cracking movement, pothole, rutting, shoving, raveling, and polished asphalt (Weng et al., 2019). From the surface condition, any remote detection is possible. Due to its capability of rapidly collecting information over wide areas, remote sensing has become an important method for evaluating the damage in infrastructure management such as road, building following major disasters. Remote sensing application for road structure or accessibility following disasters is a new field of research exploration.

The inspection method such as vibration method, vision-based method and 3D reconstruction is mostly explored. Similarly, a review by (Cao et al., 2020), reviews an image processing method, machine learning method, and 3D imaging method. A vibration-based method is a technique used in an accelerometer on vehicle platform-based for data collection (Peraka \& Biligiri, 2020). This is an advantage where it only required small storage, cost-effective, and amenable for automatic real-time data processing. However, it did not provide detailed distress condition of the roads. The pinpoint localization was also roughly geo-referenced (Mohan \& Poobal, 2017). While the 3D reconstruction method could be found in both the 3D laser scanner or photogrammetry application. Both were different in equipment use, but both methods, produced point cloud data for data mining, such as point cloud elevation, mesh or Digital Elevation Model (DEM). The detailed map using point cloud is useful however, others thing needs to be considered such as memory and computer capability. Point cloud from the laser scanner and photo-based scanner can be acquired from various platforms such as an unmanned aerial vehicle (UAV) or attach to the car for terrestrial view (Caltagirone et al., 2019; Kumar \& Angelats, 2017). Visionbased, also known as the 2D detection method was widely used by many researchers. The visionbased detection method does not require expensive equipment, additional filtering, and a training phase. Single image analysis needs deep learning implementation. Mostly image-based detection is RGB format. This detection method widely used and the advancement of image extraction analysis still ongoing. By clustering the spectral of the road, it could identify and estimate roughly the road defect. However, the need of quantifying road defect such as volume, distance and depth are important, which single image-based detection fail to supply accurately.

Objectively to get a measured image, at least two images need to be captured consecutively and overlapped. This is where the photogrammetry method applied. By this, a 3D reconstruction method has widely used and competing with a lidar-based method. A multispectral format image is a rare method used. The previous study has low resolution due to satellite platform distance (Pan et al., 2017; Pan et al., 2018). But since the micro multispectral camera invented. An applying 
multispectral camera for feature detection increased. It's been used in plant health, tree monitoring and others (Boon \& Tesfamichael, 2017). A low altitude flight for multispectral mapping emerges. Thus, this study tries to detect a road defect by using a multispectral camera in a low altitude manner. However, it is a challenge due to its factory configure setting made purposely for agriculture monitoring. By challenging the odds, this study explored the band combination to map a pothole. Fifteen combinations of the proposed bands were used with three classifiers algorithm for classification purpose. The algorithm used in this study is Maximum Likelihood, Support vector machine (SVM), and Mahalanobis distance. Therefore, this paper is provided as an intensive method to capture geometry information of pothole. The technique discusses below with comply of significant spectral band and classifier algorithm used. The analysis of geometry of the pothole is presented as an assessment.

\section{Method and study area}

The study was conducted at the Car Park area of Universiti Teknologi Mara Shah Alam, Selangor, Malaysia at $3^{\circ} 4^{\prime} 6.96 " \mathrm{~N}, 101^{\circ} 29^{\prime} 31.34 " \mathrm{E}$ (Figure 1). Potholes with a measurement of around 0.6 $\mathrm{m}$ to $1 \mathrm{~m}$ as a target feature were mapped from $10 \mathrm{~m}$ of elevation. This area was chosen as the study area because it was less congested and had good samples of potholes on site with good visibility and clear air to fly the drone. Before flying a drone, an application was made to seek permission to fly within the university's compound. The parking area was utilised by buses and trucks and it was less maintained and remained in the same condition, which was an advantage to conduct the study in situ inspection for a long-term period.

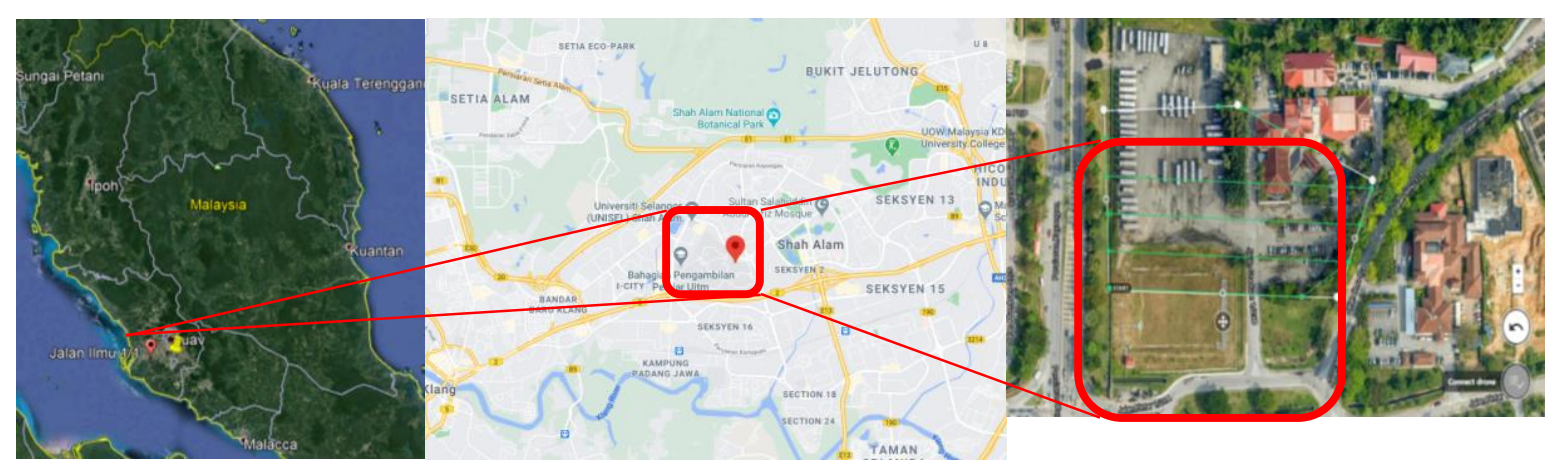

Figure 1. Car park area at the Universiti Teknologi Mara Shah Alam, Selangor, Malaysia.

Study which consisted of planning, data collection, image processing, and analysis. The planning involved site and sensor selections for this study. The data collection on site including in situ measurement and flight planning to obtain the image of potholes at the selected study areas. Image capture from $10 \mathrm{~m}$ altitude. All acquired images were processed using Pix4D, ENVI and Arcmap 10.5. Multispectral images product from pix4D were imported to ENVI for classification purpose then extracted polygon is analyse in Arcmap 10.5. Classified image were analysed using the overall accuracy and Kappa coefficient. For detail analysis on pothole geometry, error analysis study was carried out on extracted pothole area.

Pix4D have fix camera parameter of Parrot Sequoia installed in the software. Thus, no selfcalibration process carried out for Parrot Sequoia. Time lapse set up each 2 second to capture image. The reason is to ensure $70 \%$ end lap and $65 \%$ side lap for each consecutive image. The 
geometry of the pothole and asphalt. Then, all results were analysed using the Kappa coefficient and geometry error. Figure 2 illustrates the detail methodology in this study.

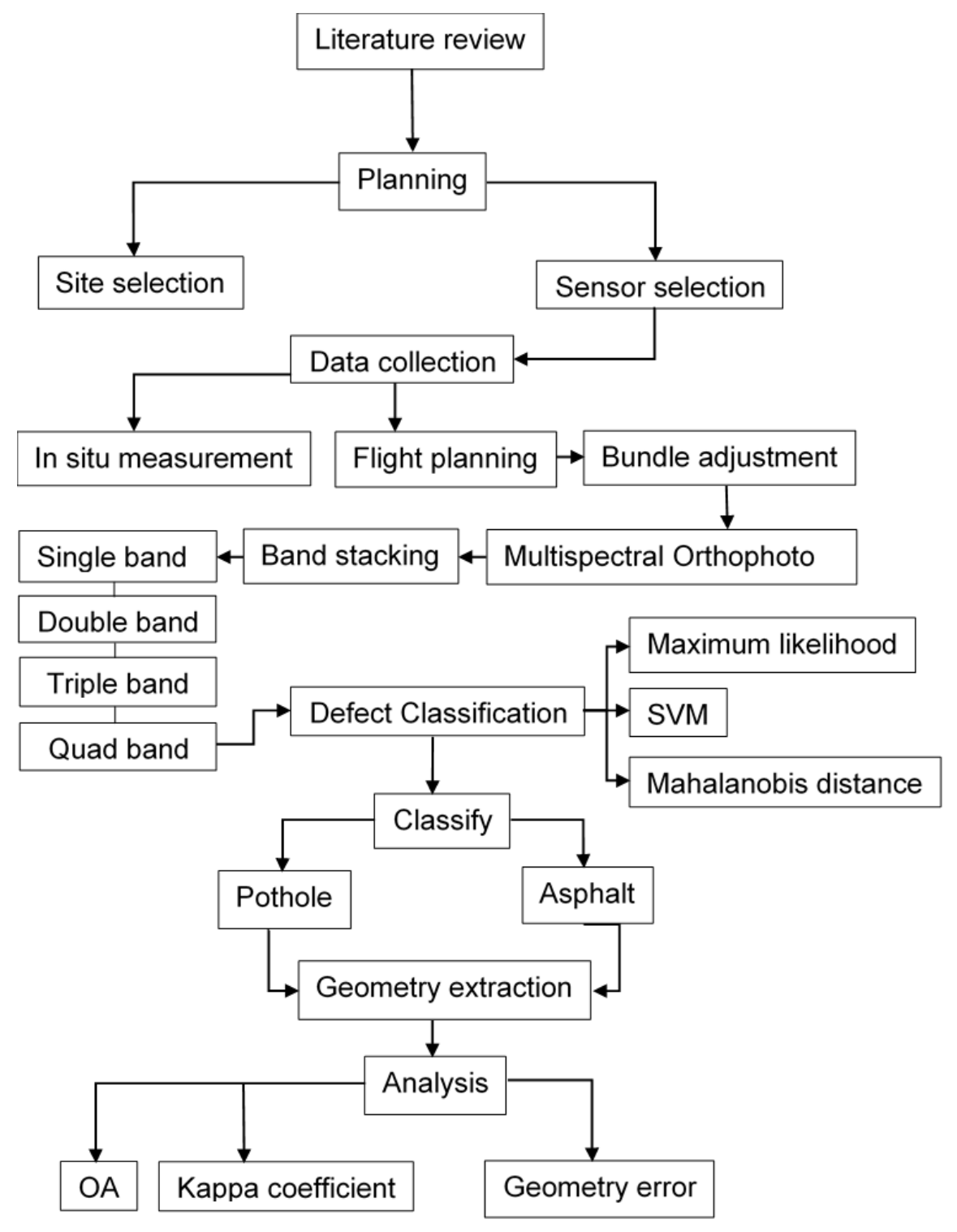

Figure 2. Detailed methodology.

A pothole is defined as a bowl-shaped depression in the pavement surface and the minimum plan dimension is $150 \mathrm{~mm}$ (Chen et al., 2020). In situ inspection on the road found two potholes around the map area, identified as pothole A and B (Figure 3). Both potholes were measured and the area was calculated for analysis purposes. This area was chosen due to the bad conditions of the road surface. Parking areas were used for buses and other heavy vehicles which operated for the students' public transportation. Lack of maintenance caused the condition of asphalt tear, and allowed water to break through it. In time the crack spread and became a pothole. 


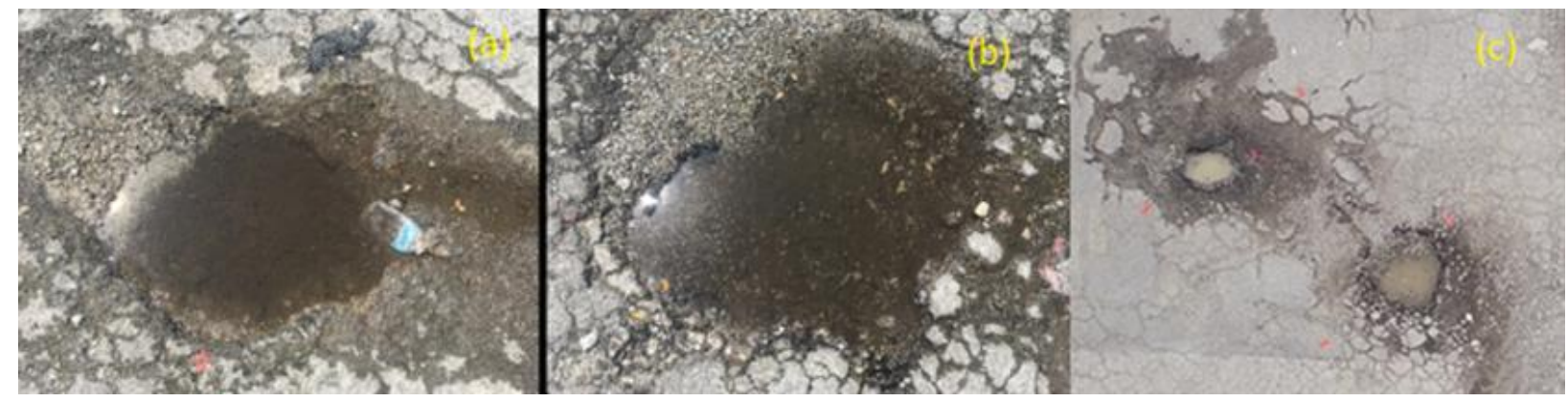

Figure 3. (a) Potholes A (b) pothole B and (c) aerial image for both potholes.

Both potholes recorded $5 \mathrm{~cm}$ in depth and the maximum pothole length was $1 \mathrm{~m}$. The Parrot Sequoia Sensor was used for detection purposes. However, a multispectral sensor was specifically used for farming/agriculture purposes. The Parrot Sequoia Sensor was designed to suit any type of drone. Thus, it is important to know the significant band on pothole features. Parrot Sequoia is specifically used in the agricultural fields with several spectral bands which measured the state of the vegetation: Green (550 nm wavelength, $40 \mathrm{~nm}$ bandwidth), Red (660 nm wavelength, $40 \mathrm{~nm}$ bandwidth), Red Edge (735 $\mathrm{nm}$ wavelength, $10 \mathrm{~nm}$ bandwidth), and Near-Infrared (790 $\mathrm{nm}$ wavelength, $40 \mathrm{~nm}$ bandwidth). In this study, the Parrot Sequoia Sensor was attached to DJI Phantom 4 to capture the images. Figure 4 illustrates the integration system between the Parrot Sequioa and Phantom 4 multirotor. Table 1 describes the multispectral sensor specification used for this study.
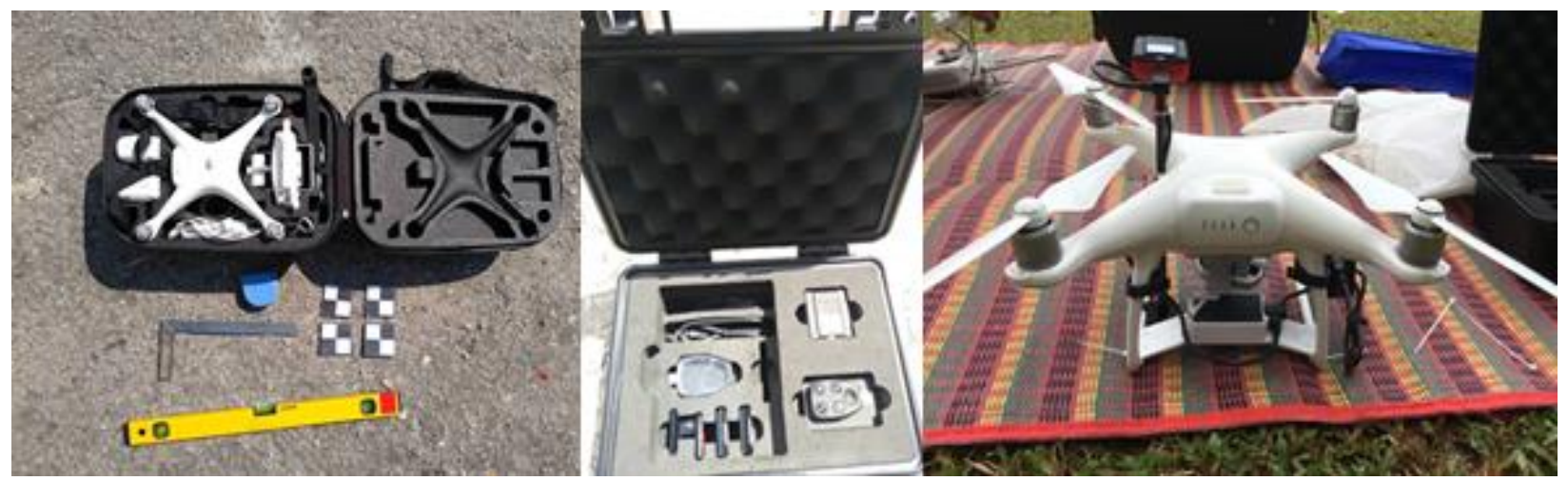

Figure 4. Parrot Sequoia Sensor mounting on DJI Phantom 4.

Table 1. Multispectral Camera specification.

\section{Camera specifications \\ Sensor \\ Multispectral sensor}

\section{Calibration}

Support

Formats Multispectral

Compatible with
Multispectral sensor + RGB camera

4-band RGB resolution $16 \mathrm{MP}, 4,608 \times 3,456 \mathrm{px}$. Single-band resolution 1.2 MP, 1,280 x 960 px Multispectral bands Green $(550 \mathrm{~nm} \pm 40 \mathrm{~nm})$ Red $(660 \mathrm{~nm} \pm 40 \mathrm{~nm})$ Red Edge $(735 \mathrm{~nm} \pm 10 \mathrm{~nm})$ Near-Infrared (790 $\mathrm{nm} \pm 40 \mathrm{~nm})$

Automatic radiometric calibration RTK/PPK

TIFF RGB JPEG eBee X, eBee SQ, eBee Classic 
The example of multispectral images from this sensor is illustrated in Figure 5. Figure 5a shows the RGB image with a resolution of about 16 megapixel, Figure 5b shows the near-infrared band image with $790 \mathrm{~nm}$ wavelength and $40 \mathrm{~nm}$ bandwidth with 1.2 megapixel resolution, Figure $5 \mathrm{c}$ shows the red edge band image with $735 \mathrm{~nm}$ wavelength and $10 \mathrm{~nm}$ bandwidth with 1.2 mega pixel resolution, Figure 5d shows the red band image with $660 \mathrm{~nm}$ wavelength and $40 \mathrm{~nm}$ bandwidth with 1.2 mega pixel resolution, Figure 5e shows the green band image with $550 \mathrm{~nm}$ wavelength and $40 \mathrm{~nm}$ bandwidth with 1.2 mega pixel resolution, and Figure $5 \mathrm{f}$ shows the orthophoto of reflectance image.

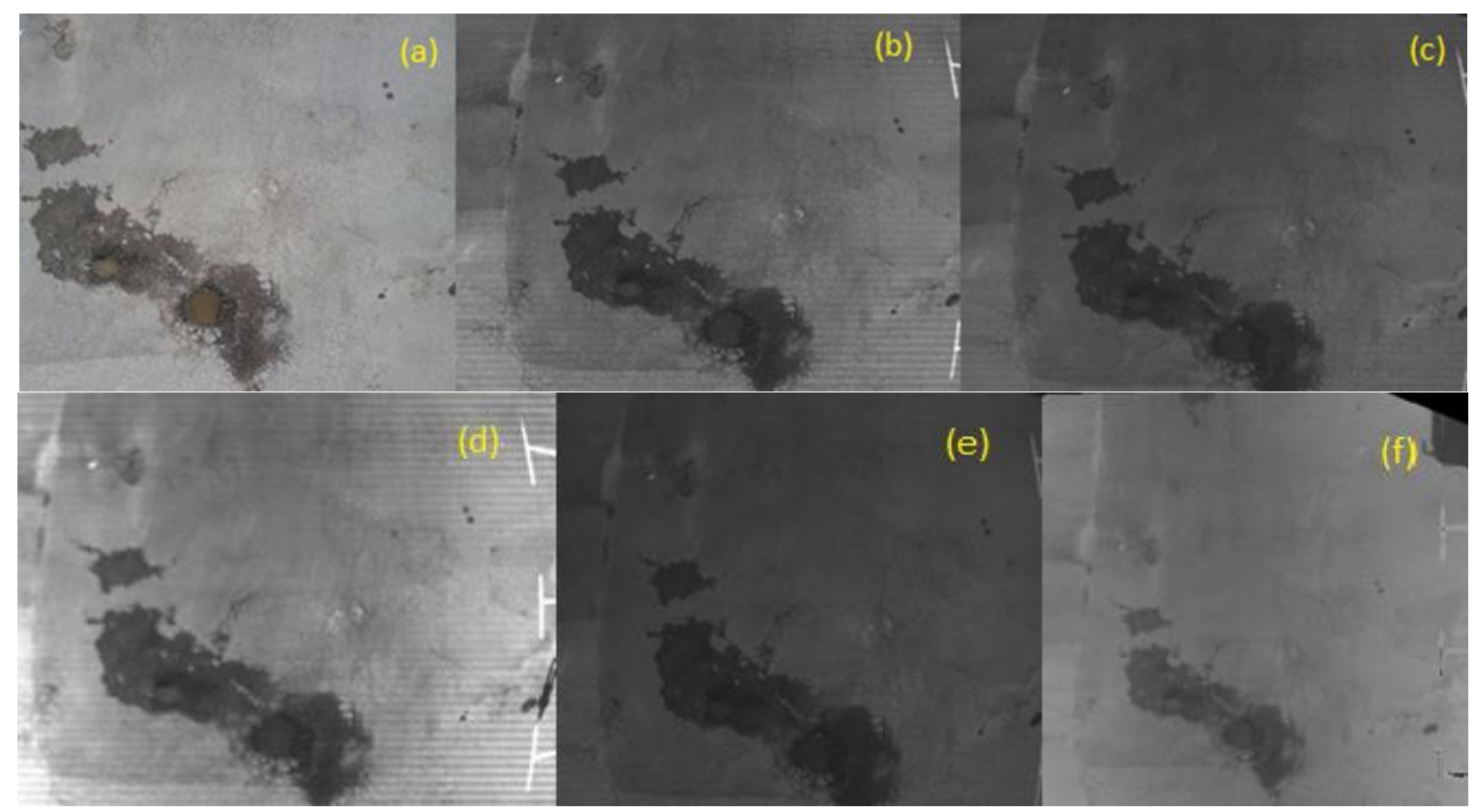

Figure 5. Multispectral Images (a) RGB; (b) Near-infrared; (c) Red-edge; (d) Red; (e) Green; (f) Orthophoto of reflectance map.

Before the flight mission, flight configuration was set-up online via Wireless Fidelity (WiFi). This study did not use the configured setting at Pix4d Mapper Capture. The available set-up for the timing of the capture could be set by a single capture, distance/GPS interval, and time lapse interval. A manual flight took place due to the low altitude at $10 \mathrm{~m}$. The reason to fly low was to ensure a good resolution for the multispectral images. The multispectral images were in the form of a TIFF File (*.tif) format. The size of the images depended on the details captured in the images, which was between 3.5 MB to 3.7 MB. The pixel of the images affected every detail in the image. Each flight could cover up to 20 minutes for 5,870 mAh (15.2V LiPo). Data was acquired and processed by Pix $4 \mathrm{~d}$ software. The output of the photogrammetry product is a reflectance map. The reflectance map was exported to the Environment for Visualising Images (ENVI) for classification of the defect areas. Three different classifiers, and 15 combination bands were proposed for this process.

Table 2 shows a band composite for this study. By dividing into sub band combinations, an analysis of the optimal number of bands for classification purposes could be conducted. The band groups were divided by the number of bands to be combined. It's consisted of a single band, double band, triple band, and quad band. All bands were tested and assessed by using the confusion 
matrix. While for the geometry assessment, all detected potholes were calculated in the area and validated with conventional measurement.

Table 2. Classifier algorithm and combination band proposed .

\begin{tabular}{|c|c|c|}
\hline CLASSIFIER & COMBINATION & BAND \\
\hline \multirow{15}{*}{$\begin{array}{c}\text { Support Vector } \\
\text { Machine, Minimum } \\
\text { Distance and } \\
\text { Parallelepiped } \\
\text { Classification }\end{array}$} & \multirow[t]{4}{*}{ Single band } & green \\
\hline & & Nir \\
\hline & & red edge \\
\hline & & red \\
\hline & \multirow[t]{6}{*}{ Double band } & green + Nir \\
\hline & & Green + red edge \\
\hline & & green + red \\
\hline & & $\mathrm{Nir}+$ red edge \\
\hline & & $\mathrm{Nir}+\mathrm{red}$ \\
\hline & & red edge + red \\
\hline & \multirow[t]{4}{*}{ Triple band } & green $+\mathrm{Nir}+$ red edge \\
\hline & & green + Nir + red \\
\hline & & $\mathrm{Nir}+$ red edge + red \\
\hline & & green + red edge + red \\
\hline & Quad band & $\begin{array}{c}\text { Green }+ \text { Nir+ red edge }+ \\
\text { red }\end{array}$ \\
\hline
\end{tabular}

\section{Results and discussion}

Detection of pothole were carried out by classification of multispectral image. A classified area of potholes was exported out for analysis purposes. Before carrying out a spatial analysis, some classifier and combination bands were opted out, because they're failed to detect a pothole. Assessment performance based from the visual condition of potholes, as shown in Figure 6. Figure $6 a$ shows an orthophoto image and Figure $6 \mathrm{~b}$ shows an undetected pothole from classifier and band combination use. While, Figure $6 \mathrm{c}$ shows a successful classification on a pothole.

Only 24 results acquired a good visual of classified pothole out of 45 results consist from 3 classifier algorithms and 15 band combinations. The analysis referred to the band combination and classifiers algorithm used in pothole detection. Figure 7 shows an error of detected pothole area. All classifier fails to detect a pothole with single band classification. At least 2 classifier algorithms success to detect a pothole with double band and triple band classification.

To identify the best classifier and band combination which can detect a pothole with a high similarity rate. A quantitative analysis was carried out. A measurement of pothole area from the conventional method is compared to a detected pothole which indicated a potential of the significant band and optimal classifier algorithm used. The lowest value of error indicated a better result of detection because it replicated the similarity of pothole geometry on the road. 


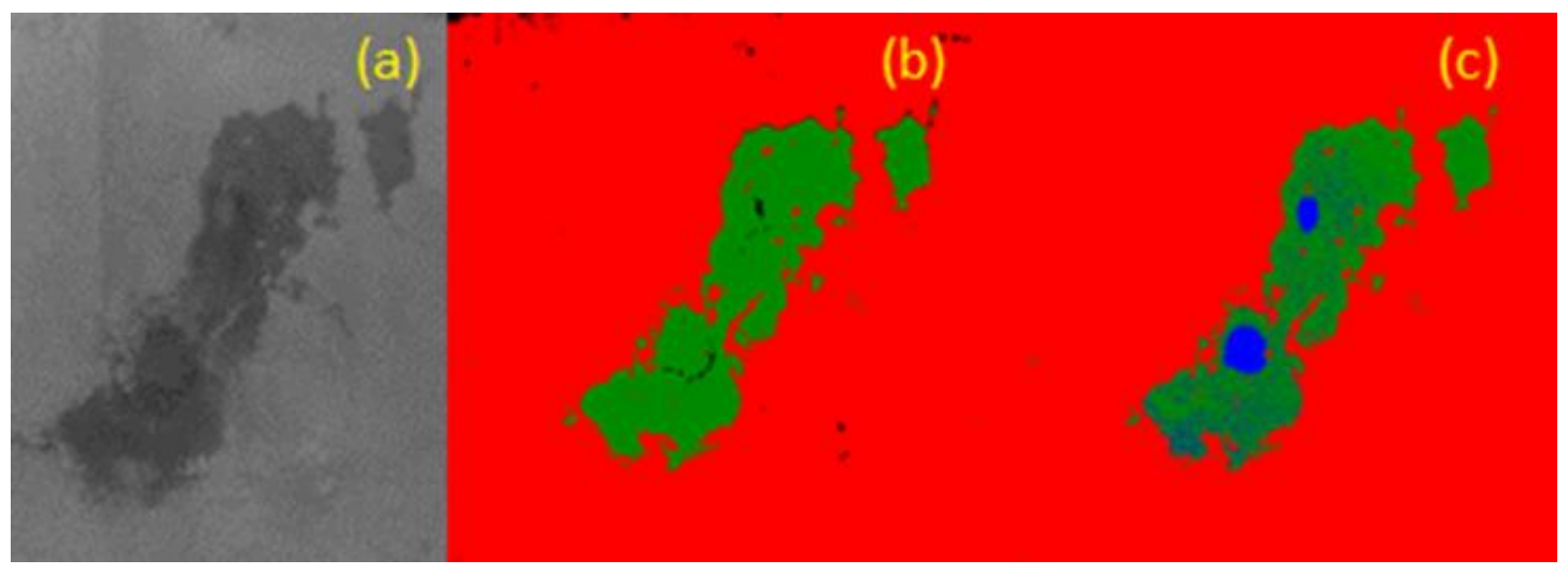

Figure 6. a) Orthophoto multispectral (b) classification of asphalt (red) and wet asphalt (green)(c) classification of pothole in blue colour.

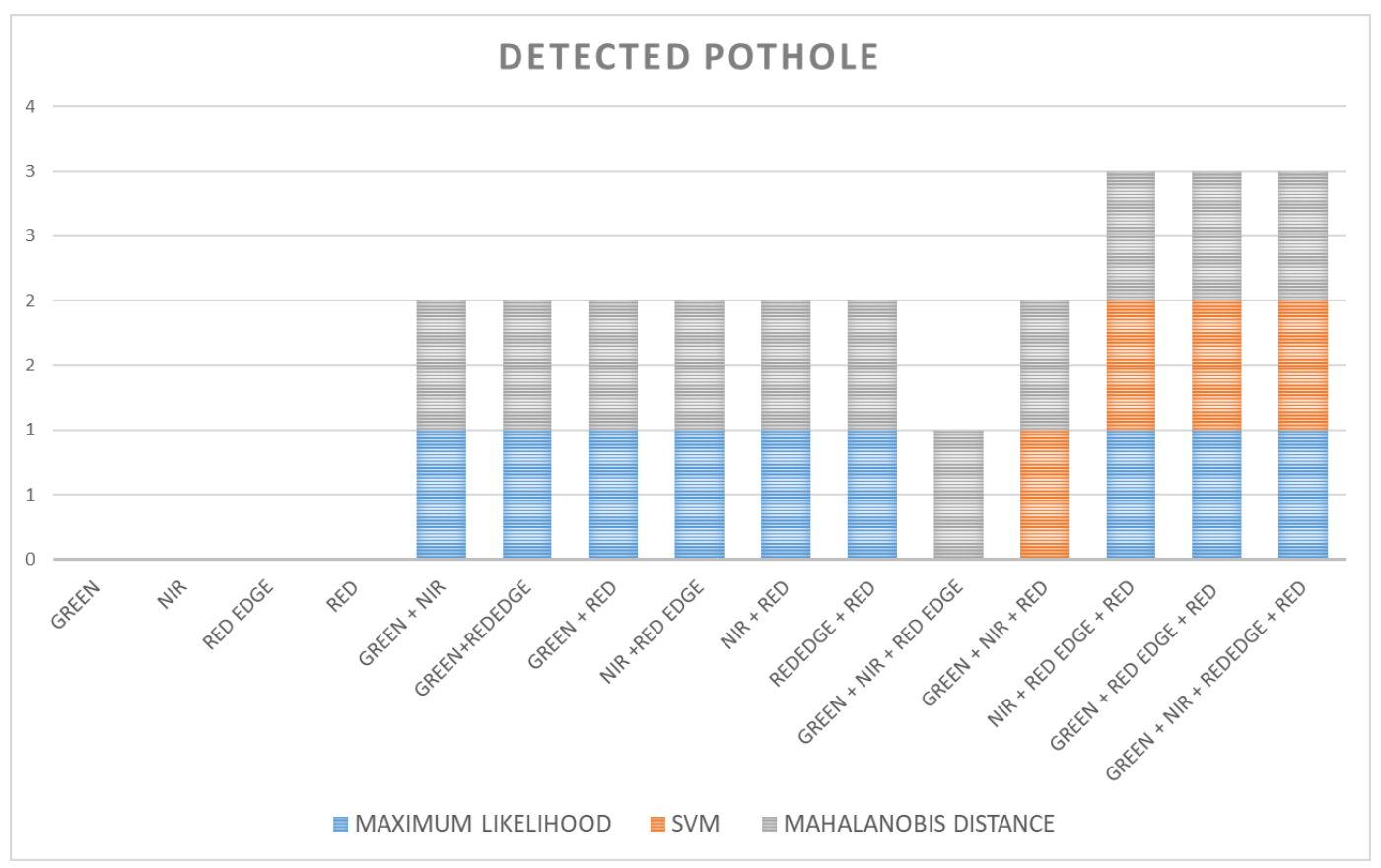

Figure 7. Performance of classifier algorithm and band combination to the detected pothole.

In situ/conventional measurements were conducted using steel tape. The measurement of the diameter of the pothole was taken to calculate the surface area. Table 3 shows a measurement of pothole diameter. An average of tenth measured diameters was used to determine the area of potholes using equation 1.

$$
\text { Area of region }=\pi r^{2}
$$

Where, $\pi$ is a constant value of 3.14159265 and $r$ is a radius of the pothole. The formula area of the region is applied in potholes $\mathrm{A}$ and $\mathrm{B}$. The method of measurement carried out was by measuring the diameter of the pothole 10 times. The average of the area is compared to the classified pothole area as shown in Table 3. Assessment of spatial determines by lowest error from both results. 
Table 3. Diameter and area of potholes.

\begin{tabular}{cccc|}
\hline \multicolumn{2}{c}{ Diameter $(\mathbf{c m})$} & \multicolumn{2}{c|}{ Area $\left(\right.$ meter $\left.^{2}\right)$} \\
\hline $\mathbf{A}$ & $\mathbf{B}$ & $\mathbf{A}$ & $\mathbf{B}$ \\
$\mathbf{8 9 . 3}$ & 70.3 & 0.626 & 0.388 \\
$\mathbf{8 6 . 3}$ & 50.9 & 0.585 & 0.203 \\
$\mathbf{9 8 . 4}$ & 58.1 & 0.760 & 0.265 \\
$\mathbf{9 7}$ & 67.4 & 0.739 & 0.357 \\
$\mathbf{9 2 . 2}$ & 64 & 0.668 & 0.322 \\
$\mathbf{1 0 2}$ & 56.9 & 0.817 & 0.254 \\
$\mathbf{9 0 . 5}$ & 48.9 & 0.643 & 0.188 \\
$\mathbf{9 6 . 9}$ & 60.5 & 0.737 & 0.287 \\
$\mathbf{9 3 . 1}$ & 71.5 & 0.681 & 0.402 \\
$\mathbf{9 4 . 8}$ & 73.1 & 0.706 & 0.420 \\
\hline & MEAN & $\mathbf{0 . 6 9 6}$ & $\mathbf{0 . 3 0 9}$ \\
\hline
\end{tabular}

Classifier algorithm analysis

Figure 8 shows a separate analysis of classifier algorithm accuracy in pothole classification. 3 classifiers were used with 15 types of band combinations in classification. The maximum likelihood and Mahalanobis distance showed a small error with double band classification, which is averagely $<0.3 \mathrm{~m}^{2}$. Double band classification indicated a small error especially for green + red edge band, green + red band, and NIR + red edge band combination. SVM shows the largest error for almost band classification combination, the error achieves $1 \mathrm{~m}^{2}$ that almost the same area of the pothole sample, thus it is an algorithm which not suitable for pothole detection. However quad band and triple band classification achieve as low as $0.6 \mathrm{~m}^{2}$.

\section{Band combination analysis}

Based on the triple band classification with mahalanobis distance and maximum likelihood, NIR + red edge + red band combination and green + red edge + red band combination showed the lowest error $<0.2 \mathrm{~m}^{2}$. While, quad band classification (green $+\mathrm{NIR}+$ red edge + red) achieves $<0.4 \mathrm{~m}^{2}$ with maximum likelihood, SVM and mahalanobis distance. All single band classification (green, NIR, red edge and red) layers failed to detect a pothole with offered classifier algorithm in this study (maximum likelihood, SVM and mahalanobis distance). Hence, the error of detected potholes is high. 

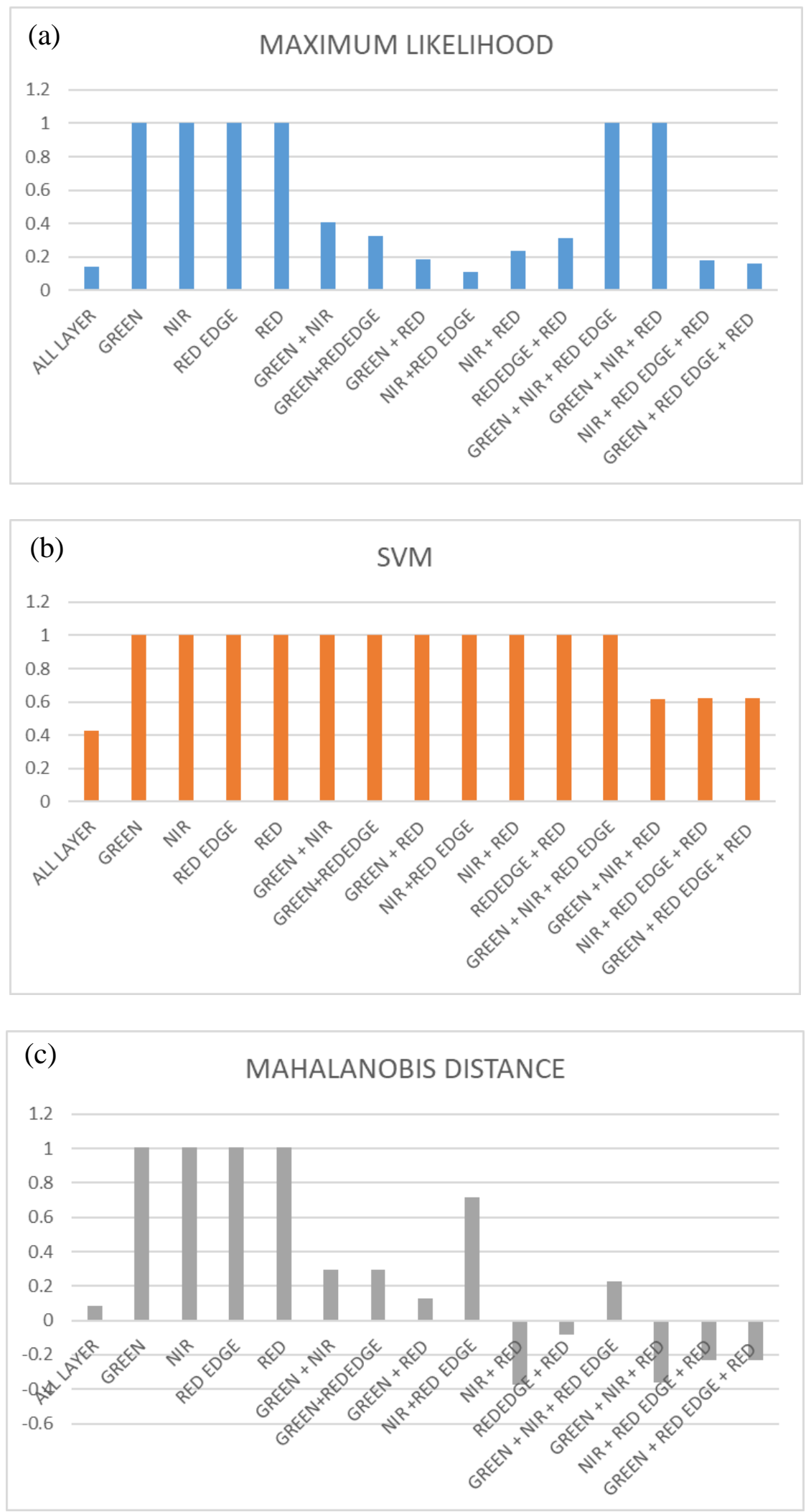

Figure 8. Detected pothole area error with (a) maximum likelihood (b) support vector machince (SVM) (c) mahalanobis distance. 


\section{Overall analysis}

Figure 9(a) shows the smallest error yield from the classifier algorithm used and Figure 9(b) shows an average of error yield from the classifier algorithm used. Classifier algorithm maximum likelihood gives a lowest error $0.108 \mathrm{~m}^{2}$ with a combination of NIR + red edge band. SVM gives the lowest error of $0.427 \mathrm{~m}^{2}$ with a combination of green $+\mathrm{NIR}+$ red edge + red band. While Mahalanobis distance gives the lowest error of $-0.082 \mathrm{~m}^{2}$ with a combination of red edge + red band. Averagely, Mahalanobis distance gives the lowest error of $0.299 \mathrm{~m}^{2}$ of all band used. Rank second, maximum likelihood with an accuracy of $0.539 \mathrm{~m}^{2}$ and SVM with an accuracy of $0.889 \mathrm{~m}^{2}$.
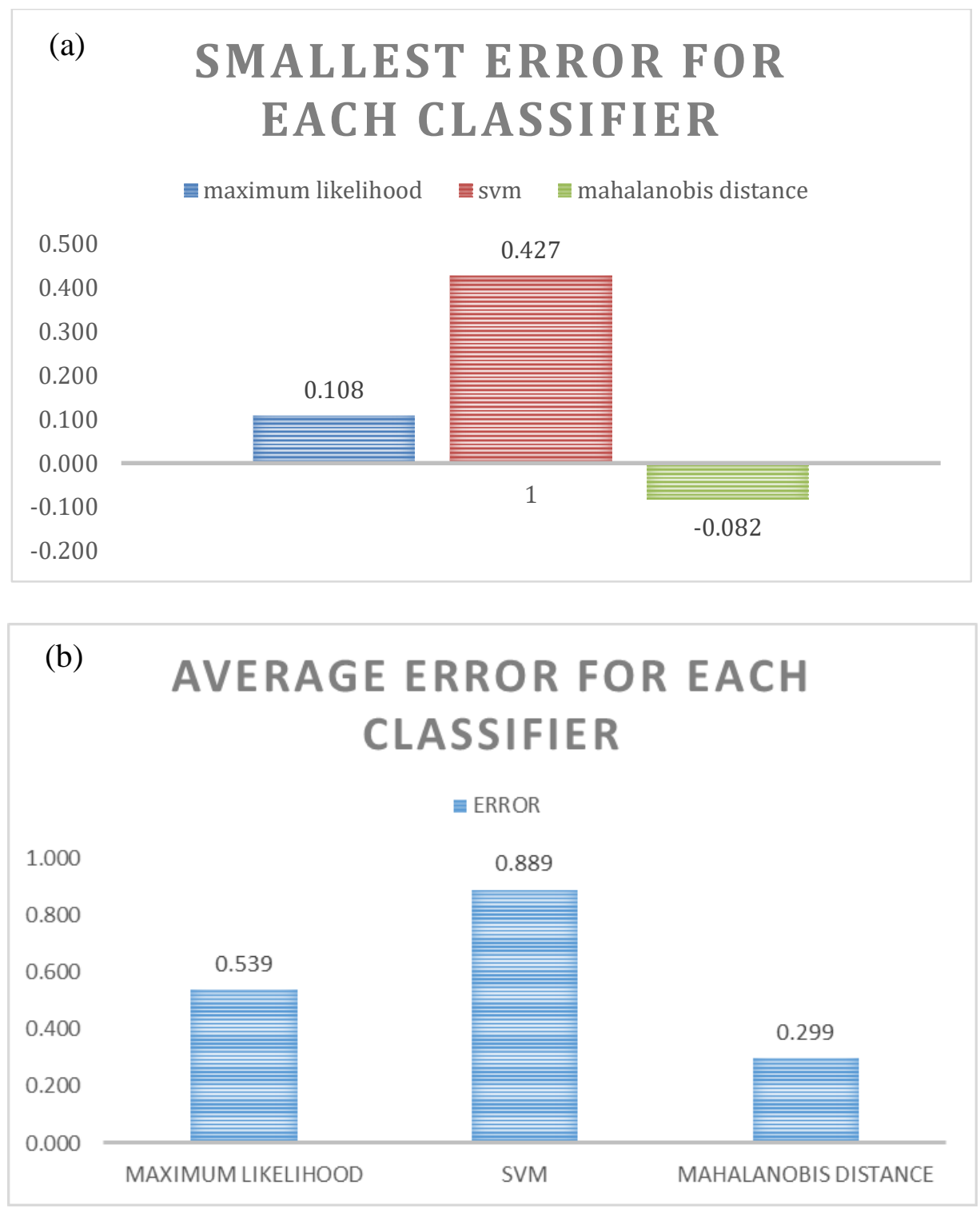

Figure 9. (a) Smallest error for each classifier (b) average error for each classifier.

The approach presented has potential on pothole detection. However, for non-tear asphalt surface of the pothole, it is impossible to be detected. This is because, a base course is the reason 
of the possible defect classification. Thus, for a small pothole or surface tears with less than 1inch/ $2.54 \mathrm{~cm}$ depth. It is a limitation to this method. As the asphalt overlays depth is around 2 inches or $5 \mathrm{~cm}$. The potential of this method is it can be used for volume determination. But it needs a digital elevation model (DEM) as surface based. The volume calculation will find volume figures based from the pothole surface to its polygon surface, which was detected early by multispectral classification. A study of this potential method will be carried not much longer.

Gao et al. (2020) has proposed the method Library for support vector machine (LIBSVM) to extract potholes on the cement concrete pavement. This study also used different classifiers such as otsu, edge detection, K-mean and watershed algorithm. Based on the results, the proposed method has less time in segmentation image processing compared to other classifiers. The proposed method also has better segmentation for pothole extraction. However, they did not discuss on the accuracy of results in this study. Wang et al. (2017) has segmented the pothole by Markov random field model and achieved an accuracy $85 \%$ of the extracted pothole. Therefore, this study also achieves similar results with the previous studies but using a different approach such as maximum likelihood, support vector machine and mahalanobis distance. The mahalanobis gave a small error compared to other classifiers. However, this study also investigates the effect on band combinations in multispectral sensors and it was found that, the band combination between red edge and red gives the lowest error compared to other band combinations.

\section{Conclusion}

The best companion of band combination for classification and classifier algorithm suggest in this study is NIR + red edge band with maximum likelihood, green + NIR + red edge + red band with SVM and red edge + red band with Mahalanobis distance. The best classifier algorithm with the lowest mean error for all band combinations used in the classification of a pothole is Mahalanobis distance. The most perform and the prominent band for pothole detection is red edge and red as it appears frequently in the lowest error assessment. It is a pleasure to include a blue band for further research. The existence of water content inside the potholes which absorbed into the subgrade level of the pavement, could easily be detected. Hypothetically, it could increase the accuracy of measurement for classification. Another idea to upgrade the detection of potholes is by using Object-based image analysis or machine learning applications. In terms of the accuracy aspect, a need to understand the multiscale effect of the detection process is compulsory. The lower resolution of imagery limits the potential of the Parrot Sequoia image. It was built for agriculture purposes, perhaps it was not considered an accurate aspect for farming. Lastly, acquired images from different parameters with different areas, and to understand the proportion effect into pothole extraction quality.

\section{Acknowledgement}

Faculty of Architecture, Planning, and Surveying Universiti Teknologi MARA (UiTM), Research Management Centre (RMC) and Ministry of Higher Education (MOHE) are greatly acknowledged for providing the fund GPK grant 600-RMC/GPK 5/3 (223/2020) to enable this research to be carried out. The authors would also like to thank the people who were directly or indirectly involved in this research. 


\section{References}

Akagic, A., Buza, E. Omanovic, S. \& Karabegovic , A. (2018). Pavement crack detection using Otsu thresholding for image segmentation. 2018 41st International Convention on Information and Communication Technology, Electronics and Microelectronics (MIPRO), 1092-1097

Boon, M. A., \& Tesfamichael, S. (2017). Wetland vegetation integrity assessment with low altitude multispectral UAV imagery. International Archives of the Photogrammetry, Remote Sensing and Spatial Information Sciences - ISPRS Archives, 42(2W6), 55-62. https://doi.org/10.5194/isprs-archives-XLII-2-W6-55-2017

Caltagirone, L., Bellone, M., Svensson, L. \& Wahde, M. (2019). LIDAR-camera fusion for road detection using fully convolutional neural networks. Robotics and Autonomous Systems. 111, 125-131. https://doi.org/10.1016/j.robot.2018.11.002

Cao, W., Liu, Q., \& He, Z. (2020). Review of Pavement Defect Detection Methods. IEEE Access, 8, 14531-14544. https://doi.org/10.1109/ACCESS.2020.2966881

Chen, H., Yao, M. \& Gu, Q. (2020). Pothole detection using location-aware convolutional neural networks \}, International Journal of Machine Learning and Cybernetics, 11, 899-911.

Gao, M., Wang, X., Zhu, S.\& Guan, P. (2020). Detection and Segmentation of Cement Concrete Pavement Pothole Based on Image Processing Technology. Mathematical Problem in Engineeering. 2020, 1-13.

Gandhi, J.R., Jaliya, U.K., \& Thakore, D. (2019). A Review Paper on Pothole Detection Methods. International Journal Of Computer Sciences And Engineering, 7(2), 379-383.

Iseli, C., \& Lucieer, A. (2019). Tree species classification based on 3D spectral point clouds and orthomosaics acquired by snapshot hyperspectral UAS sensor. XLII(June), 10-14.

Kumar, P. and Angelats, E. (2017). An Automated Road Roughness Detection From Mobile Laser Scanning Data, Int. Arch. Photogramm. Remote Sens. Spatial Inf. Sci., XLII-1/W1, 91-96, https://doi.org/10.5194/isprs-archives-XLII-1-W1-91-2017.

Mohan, A., \& Poobal, S. (2017). Crack detection using image processing: A critical review and analysis. Alexandria Engineering Journal, 57, 787-798.

Noi, P. T., \& Kappas, M. (2018). Comparison of random forest, k-nearest neighbor, and support vector machine classifiers for land cover classification using Sentinel-2 imagery. Sensors (Switzerland), 18(1). https://doi.org/10.3390/s18010018

Pan, Y., Zhang, X., Sun, M., \& Zhao, Q. (2017). Object-based and supervised detection of potholes and cracks from the pavement images acquired by UAV. International Archives of the Photogrammetry, Remote Sensing and Spatial Information Sciences - ISPRS Archives, 42(4W4), 209-217. https://doi.org/10.5194/isprs-archives-XLII-4-W4-209-2017

Pan, Yifan, Zhang, X., Cervone, G., \& Yang, L. (2018). Detection of Asphalt Pavement Potholes and Cracks Based on the Unmanned Aerial Vehicle Multispectral Imagery. IEEE Journal of Selected Topics in Applied Earth Observations and Remote Sensing, 11(10), 3701-3712. https://doi.org/10.1109/JSTARS.2018.2865528

Peraka, N.S.P. \& Biligiri, K.P. (2020). Pavement asset management systems and technologies: A review. Automation in Construction, 119, 103336

Schnebele, E., Tanyu, B.F., Cervone, G. \& Waters, N. (2015). Review of remote sensing methodologies for pavement management and assessment. Eur. Transp. Res. Rev. 7, 7 (2015). https://doi.org/10.1007/s12544-015-0156-6

Wang, P., Hu, Y., Dai, Y., \& Tian, M. (2017). Asphalt Pavement Pothole Detection and 
Segmentation Based on Wavelet Energy Field. Mathematical Problem in Engineering. 2017, 1-13.

Weng, X., Huang, Y. \& Wang, W. (2019). Segment-based pavement crack quantification. Automation in Construction, 105, 102819

Wolf, J., Discher, S., Masopust, L., Schulz, S., \& Richter, R. (2018). Combined Visual Exploration Of $2 d$ Ground Radar And 3d Point Cloud Data For Road Environments. XLII(October), $1-2$.

Zhang, J., Zhang, J., \& Zhao, Z. (2018). Study on the classification of Gaofen-3 polarimetric SAR images using deep neural network. International Archives of the Photogrammetry, Remote Sensing and Spatial Information Sciences - ISPRS Archives, 42(3), 2263-2266. https://doi.org/10.5194/isprs-archives-XLII-3-2263-2018 